\title{
Methodology and Design of a National Epidemiological Study on Adult Neuromuscular Disease
}

\author{
Stela Lefter ${ }^{a, b}$ Orla Hardiman ${ }^{b}$ Aisling M. Ryan ${ }^{a}$ \\ ${ }^{a}$ Department of Neurology, Cork University Hospital, Cork and bepartment of Neurology, Beaumont Hospital, \\ Dublin, Ireland
}

\section{Key Words}

Neuromuscular diseases · Epidemiology · Research methods

\begin{abstract}
Background: There have been no previous populationbased studies of adult neuromuscular disease (NMD) in the Republic of Ireland (Rol). This article describes methods and case-ascertainment strategies used to identify patients with inherited and acquired NMD for the purpose of obtaining the prevalence of these disorders in the Rol. Methods: This epidemiological study was conducted between January 2012 and January 2014. Prospective and retrospective (until 1990) case identification of adults with inherited and acquired NMD have been carried out. Multiple countrywide patient identification sources including neuromuscular clinics, hospital neurology databases, the hospital in-patient enquiry (HIPE) system of each hospital and the records of Muscular Dystrophy Ireland, a nonprofit organisation, were used. Results: In total, 3,724 potential cases were identified. Of these, 1,083 were excluded because 869 cases represented duplicates or triplicates, 133 were coded incorrectly in HIPE, 74 patients were deceased and 7 patients had moved out of
\end{abstract}

the country. The highest number of cases was identified in neurology databases and HIPE (1,724 and 884, respectively). A total of 2,641 individuals fulfilled the inclusion criteria and were included in the study. Conclusion: Detailed epidemiological data of this nature is difficult to acquire in the current structure of the Irish health service, requiring multiple sources including input from voluntary patient organisations. The development of a national patient registry for some or all of these conditions would greatly facilitate standardised data recording, giving a true picture of the burden of neuromuscular diseases in a population.

๑ 2014 S. Karger AG, Basel

\section{Introduction}

Neuromuscular diseases (NMD) are a complex group of heterogenous disorders, which can be broadly divided into disorders affecting the anterior horn cells, peripheral nerves, neuromuscular junctions and muscle [1]. A few studies looked at both acquired and inherited NMD in children $[2,3]$, but a literature review has shown no such study in the adult population. Epidemiological studies that include all types of NMD are very rare, whereas

\section{KARGER}

E-Mail karger@karger.com

www.karger.com/ned
(C) 2014 S. Karger AG, Basel

0251-5350/14/0432-0123\$39.50/0
Dr. Stela Lefter

Neuromuscular Research Fellow, Department of Neurology

Cork University Hospital

Cork (Ireland)

E-Mail stela_lefter@yahoo.com 
single-disorder studies are much more common [4-11]. Many patients with NMD have similar requirements in terms of access to expertise for diagnostic (neurologists, neurophysiologists) and treatment modalities (orthopaedics, orthotics, physiotherapy, cardiac and respiratory input, etc.). Investigating the NMD group as a whole thus gives a better indication of the burden of these conditions and a better pointer to the services required to meet the needs of these patients.

The 6 counties of Northern Ireland (NI) and the 26 counties of Republic of Ireland (RoI) encompass all of the island of Ireland. A previous study analysed the prevalence of inherited NMD in all age groups in Northern Ireland (NI), which has similar demographics to the RoI [12]. The NI data does provide a guide to the likely overall prevalence (total population) of inherited NMD in RoI. However, the NI study included the paediatric population and did not include acquired conditions.

Neurology patients in RoI are looked after by neurologists working in the public and private health care sectors. Fifteen public hospitals, and 8 private hospitals and clinics function as neurology referral centres in the country. Dedicated neuromuscular clinics in two tertiary referral hospitals - Beaumont Hospital, Dublin and Cork University Hospital, Cork - serve as specialised referral centres for patients with NMD in RoI. These two tertiary referral hospitals have neuropathology departments serving the whole country's population. However, not all neuromuscular patients are referred to or followed up in these 2 specialised neuromuscular clinics. A significant number are followed up by neurologists locally in the public or private hospitals in their own catchment area.

In this article, we describe in detail the methods used to identify patients with NMD in the RoI.

\section{Materials and Methods}

Case ascertainment commenced in January 2012 and was completed in January 2014. Ethical approval was initially established for the two tertiary referral centres (Beaumont Hospital and Cork University Hospital). Subsequently, individual ethical approval, where required, was obtained from the ethics committee of each participating institution.

\section{Study Population}

The study focused on the adult population of RoI, a geographically well-defined area comprising 26 counties (online suppl. fig. E-1; for all online suppl. material, see www.karger.com/doi/ $10.1159 / 000367634)$ of the island of Ireland. The estimated total population is $4,588,252$ of which adults ( $\geq 18$ years) is $3,439,565$, based on the 2011 RoI Census 2011 (Central Statistics Office Ireland) [13].
Inclusion and Exclusion Criteria

Inclusion and exclusion criteria are illustrated in table 1.

\section{Diagnostic Criteria}

Diagnostic criteria for inclusion and classification were the following: for chronic inflammatory demyelinating polyneuropathy (CIDP), those proposed by the European Federation of Neurological Societies and the Peripheral Nerve Society (EFNS/PNS) published in 2006 and updated in 2010 [14]; for acute inflammatory demyelinating polyradiculoneuropathy (AIDP), those described by Asbury [15]; for inherited neuropathies and inherited muscle disease, those established by the European Neuromuscular Centre in 1998 [16] and from more recent key references for individual conditions as proposed in the 2013 version of the gene table of monogenic neuromuscular disorders (nuclear genome) [17]. Diagnostic standards for congenital myasthenic syndrome were those described by Kinali [18]. Diagnosis of non-dystrophic myotonia and periodic paralysis was established by clinical, electrophysiologic features and genetic confirmation. Diagnosis of myasthenia gravis (MG) was established by history and examination features with the result of at least 1 positive paraclinical test (antibody, electrophysiology or edrophonium). Lambert-Eaton myasthenic syndrome was established by demonstrating characteristic electrophysiologic features (idiopathic form) with additional evidence of an underlying neoplasm (paraneoplastic form) and supported by voltage-gated calcium channel antibodies. Diagnosis of sporadic inclusion body myositis (sIBM) was based on Modified IBM criteria from Medical Research Council Centre for Neuromuscular Diseases 2010 [19].

\section{Case Ascertainment Sources}

A neuromuscular research fellow (SL, first author) conducted the study working jointly between the two dedicated neuromuscular centres in RoI (Cork University Hospital and Beaumont Hospital, Dublin). All patients recruited through neuromuscular clinics had a full neurological examination and their medical notes and investigations/results were reviewed by one of the investigators. The research fellow also searched for cases in all neurology databases of 14 public hospitals (out of a total of 15) and 7 private hospitals/clinics (out of a total of 8), 2 neuropathology departments and 5 neurophysiology departments.

In order to aid recruitment, multiple methods were used to increase awareness of this study with a view to achieving as complete ascertainment as possible. All adult and paediatric neurologists, neurophysiologists, neuropathologists, respiratory physicians, cardiologists and county physicians were notified in writing regarding the study aims. Referrals by these physicians of existing neuromuscular patients or patients suspected of exhibiting features of NMD to the two neuromuscular clinics were encouraged. General practitioners were notified about the study by an advertisement article in the Irish College of General Practitioners national journal. The fellow presented the project at the national neurology meetings and neurology intradepartmental educational meetings in hospitals across the country. The study was advertised in local medical newspapers. The patient voluntary organization Muscular Dystrophy Ireland (MDI) informed all their members about the study and invited them to participate by a correspondence to each member and study advertisement on their website (www.mdi.ie). The fellow was freely contactable by people who might wish to be enrolled in the study and by all physicians by 
Table 1. Study inclusion and exclusion criteria

Inclusion criteria

Adults ( $\geq 18$ years) living in the Republic of Ireland $\geq 5$ years with the following conditions:

(1) Neuropathy

Acquired inflammatory demyelinating neuropathies (e.g., GBS, CIDP, MMN, PDN)

Inherited neuropathy (e.g., CMT, HNPP, dHMN, HMSN 5, FAP)

Spinal muscular atrophy (types 3 and 4 )

Kennedy's disease

Neuromyotonia

(2) Neuromuscular Transmission Disorder

Myasthenia Gravis

Lambert-Eaton myasthenic syndrome

Congenital myasthenic syndrome

(3) Muscle disease

Muscular dystrophies (e.g., DMD, BMD, LGMD, FSHMD, myotonic dystrophy)

Distal myopathy, myofibrillar myopathy

Congenital myopathy

Muscle channelopathy (myotonia congenita, paramyotonia congenita, periodic paralysis, ATS)

Metabolic myopathy (glycogen storage, fatty acid and mitochondrial)

Inclusion body myositis, macrophagic myofasciitis

Exclusion criteria

Under 18 years of age

(1) Acquired axonal neuropathy

(2) Myalgia without muscle weakness

(3) Polymyositis, Dermatomyositis

(4) Post-Polio syndrome

GBS = Guillain-Barré syndrome; CIDP = chronic inflammatory demyelinating polyneuropathy; MMN = multifocal motor neuropathy; $\mathrm{PDN}$ = paraproteinaemic demyelinating neuropathy; CMT = Charcot-Marie-Tooth disease; HNPP = hereditary neuropathy with liability to pressure palsies; $\mathrm{dHMN}=$ distal hereditary motor neuropathy; HMSN = hereditary motor sensory neuropathy; FAP = familial amyloid polyneuropathy; DMD = duchenne muscular dystrophy; BMD = decker muscular dystrophy; LGMD = limb girdle muscular dystrophy; FSHMD = facio-scapulo-humeral muscular dystrophy; ATS = andersen-tawil syndrome.

means of a dedicated mobile phone and email address. The fellow presented the project at multiple patient-oriented meetings organised by MDI during a two-year study period. Permission was granted to search the MDI database. Patients identified via MDI were recruited by referring to one of the neuromuscular clinics or by arranging a review by the fellow in the MDI office.

The hospital in-patient enquiry (HIPE) is a national computerbased database system designed to collect demographic, clinical and administrative data on discharges from acute public sector hospitals nationally in RoI [20]. This was searched in four major adult hospitals for discharges from 1st January 1990 until 31st August 2013 with diagnosis under the following ICD codes: ICD-9-CM: 3351033511, 3560-3562, 3570, 3578, 3580-3581, 3588, 3590-3592, 3598, 3596 and ICD-10-AM: G121, G129, G600, G610, G618, G708, G700, G702, G710-G713, G736, G724. Cases identified through HIPE system were crosschecked with patient medical notes for diagnostic verification and further disease classification purposes. For patients identified from the HIPE system and neurology databases, supportive data from preceding investigations in the course of diagnostic evaluation were used where possible.

The following demographic variables were recorded: full name, date of birth, gender, hospital number, patient address, country of birth, name of the treating neurologist, name of the hospital that the patient is attending, source/sources where patient was ascertained. The NMD group (e.g., acquired neuropathy, inherited neuropathy, neuromuscular junction disorder, muscular dystrophy, skeletal muscle channelopathy, acquired muscle disease) followed by more specific diagnosis (e.g., AIDP, CIDP, CMT, myasthenia gravis, limb girdle muscular dystrophy, periodic paralysis, myotonia congenital, sIBM) were recorded. Where available the precise genetic or other (e.g., immune-mediated) diagnosis was recorded (99 entities). The following relevant diagnostic investigations were obtained: for AIDP - neurophysiology, cerebrospinal fluid protein level, ganglioside antibodies; for CIDP - neurophysiology, cerebrospinal fluid protein level, ganglioside antibodies (GM1), anti MAG, serum paraproteins and where available sural nerve biopsy; for inherited neuropathies - neurophysiology and where available genetic tests; for neuromuscular junction disorders - relevant autoantibodies, neurophysiology, Tensilon test, result of thoracic computer tomography; for genetic muscle disease - creatine kinase, neurophysiology, muscle biopsy and where available genetic tests. In cases where the diagnosis from different sources did not match (this often occurred when a diagnosis given years ago was later changed as a result of more recent diagnostic tests), the most 
Fig. 1. Venn diagram demonstrating sources overlap. HIPE = Hospital in-patient enquiry; MDI = Muscular Dystrophy Ireland; GBS = Guillain-Barré syndrome (GBS Society).

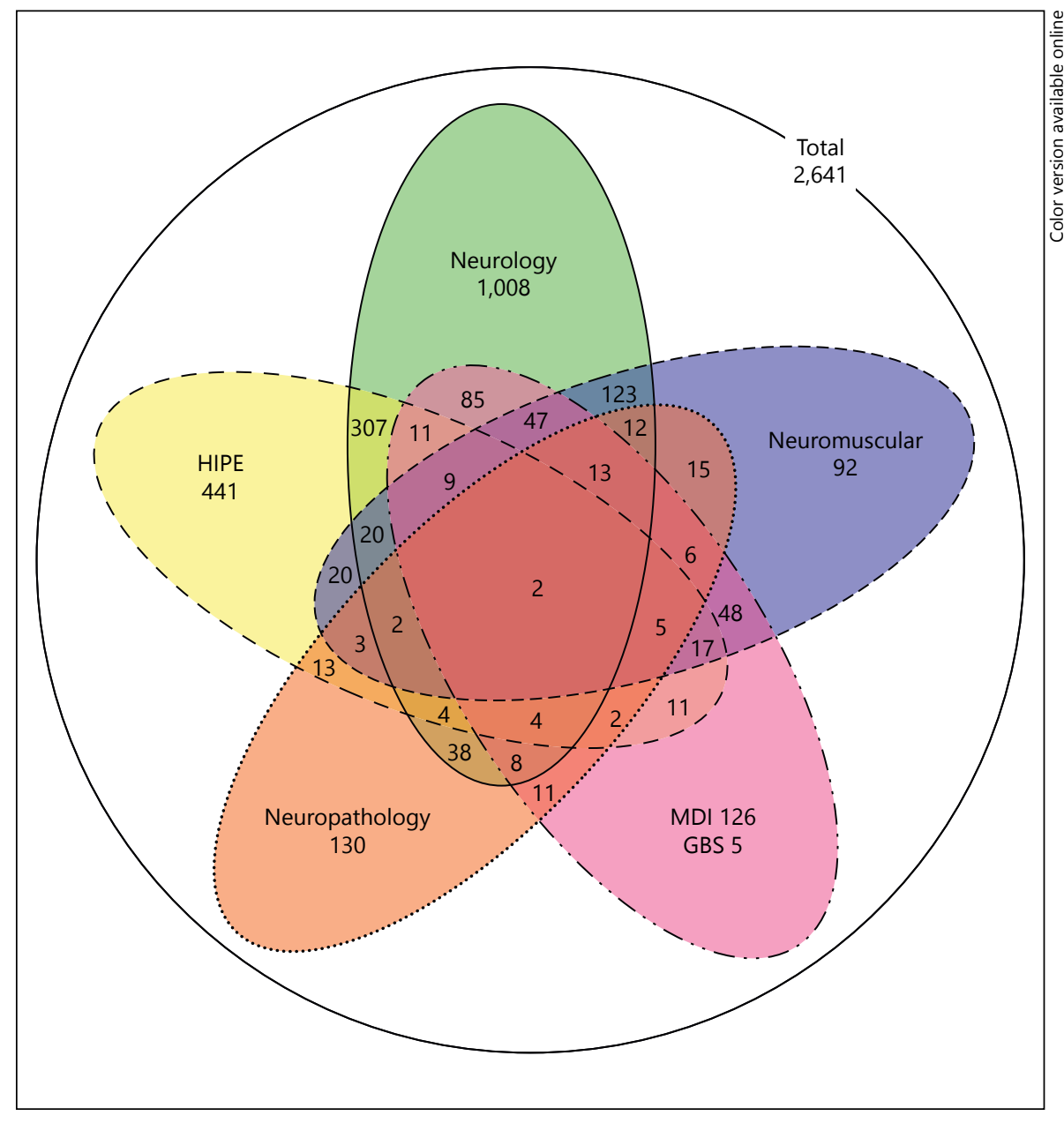

Table 2. Cases identified in each source

\begin{tabular}{lcc}
\hline All NMD & & \\
\hline source & number of cases (\%) & unique to source \\
\hline Neurology/Neurophysiology & $1,724(46.3)$ & 1,008 \\
Neuromuscular clinics & $434(11.6)$ & 92 \\
Neuropathology & $269(7.2)$ & 130 \\
HIPE & $884(23.7)$ & 441 \\
MDI & $406(10.9)$ & 126 \\
GBS society & $7(0.2)$ & 5 \\
\hline
\end{tabular}

recent diagnosis was used. Data from all perused sources was crosschecked to eliminate duplicates. In cases where one of the variables was missing (e.g., date of birth), other available variables such as patient name, diagnosis and address were used for linking in order to prevent duplicates.

All patients enrolled, at some stage in their illness, had to be seen and diagnosed with NMD by a neurologist. Furthermore, in every case - in order to be included in the study - there had to be at least one abnormal investigation (e.g., laboratory, neurophysiology or histopathology). Where patients were labelled as 'possible' myopathy or neuropathy or the diagnosis was otherwise unclear, these patients were not included in the study. Similarly, where patients were undergoing investigation and a diagnosis had not yet been reached, these cases were not included.

Identified individuals were classified according to the ascertained source into 6 groups (table 2). Subjects were further subdivided according to whether they were found uniquely in one source or were identified in multiple overlapping sources (fig. 1).

Descriptive statistical analysis were performed using IBM SPSS Statistics v20 (@ SPSS 2010 Inc.) and Microsoft Excel (@ 2010 Microsoft Inc.).

\section{Results}

A flow diagram of identification of potential cases with NMD is illustrated in figure 2. A total number of 3,724 potential cases were identified from 6 sources. Out of these 3,724 cases, 869 cases were also identified in anoth- 


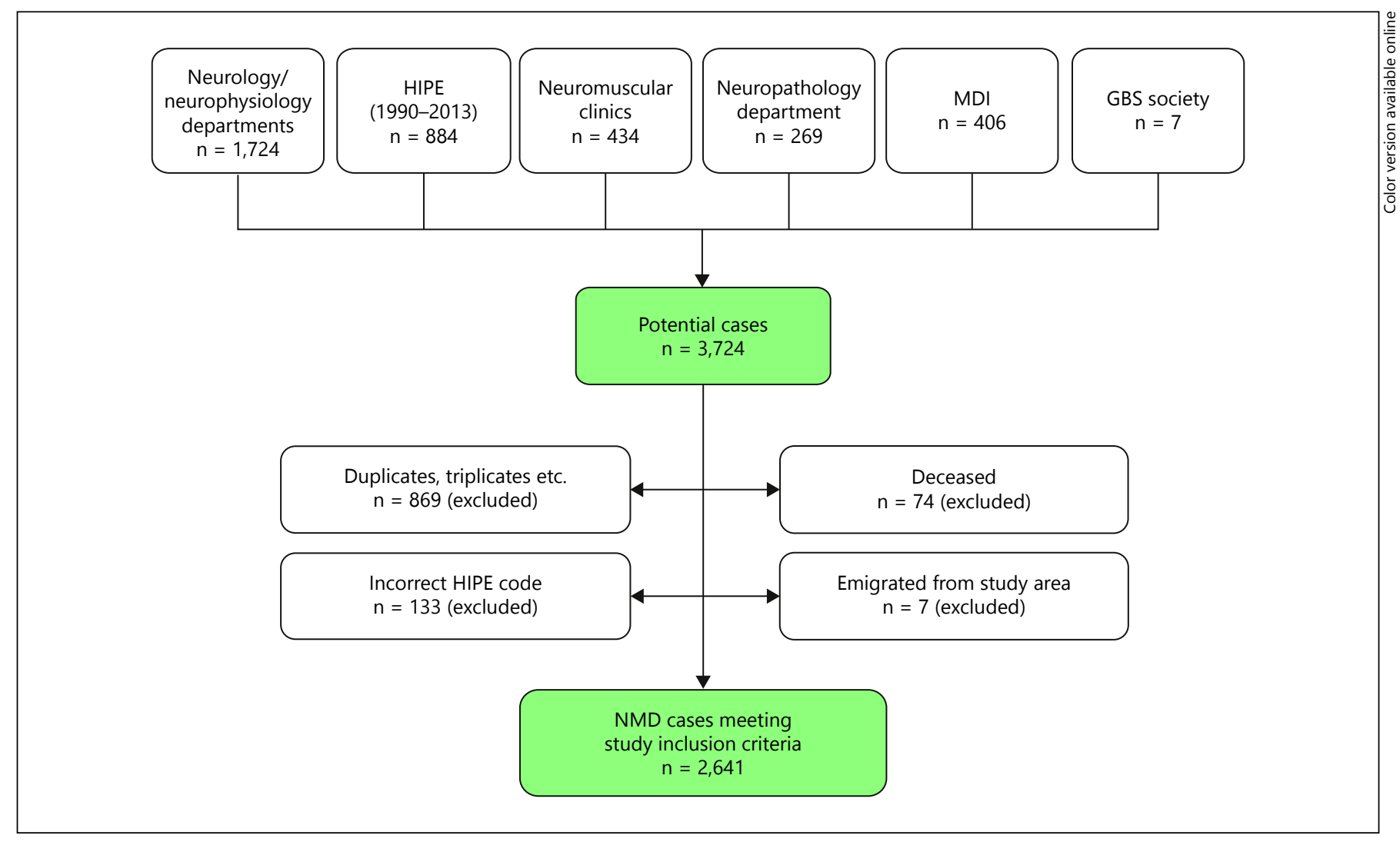

Fig. 2. Flow diagram of identification of potential cases of neuromuscular disorders in RoI, 1990-2013. HIPE = Hospital in-patient enquiry; MDI = Muscular Dystrophy Ireland; GBS = Guillain-Barré syndrome.

er source or sources (i.e., duplicates, triplicates), and these were excluded from the study. HIPE analysis was carried out in 4 hospitals: 884 cases were identified and their medical charts were retrieved for diagnosis crosscheck and review of supporting investigations. After chart review, 133 were found to have been coded incorrectly and these were excluded; 121 (91\%) of these 133 cases were coded in one hospital where the vast majority - 115 cases (95\%) - were coded erroneously as hereditary sensory neuropathy (ICD 9: 3562). The correct diagnoses of patients with these neuropathies ranged from idiopathic, diabetic, and uraemic to other acquired neuropathies. Furthermore, 74 patients were deceased and 7 patients had moved out of the country and these were also excluded. This resulted in 2,641 as the actual number of cases recruited for inclusion in this study.

The highest number of total cases and 'unique to source' cases were identified in neurology departments and HIPE. A Venn diagram illustrating the sources overlap is seen in figure 2; 307 cases were identified in HIPE and neurology departments only, 123 in both neurology

Methodology and Design of a National Epidemiological Study on Adult NMD departments and neuromuscular clinics only, 85 in neurology departments and MDI only, 48 in MDI and neuromuscular clinics.

A total of 2,641 individuals fulfilling the inclusion criteria were identified. 1,481 (56.1\%) were males and 1,160 (43.9\%) females. The male:female ratio was 1.28:1. Age was calculated as per 31st December 2013. The age was missing in 150 cases ( 81 males and 69 females). The overall age range was $18-93$ years with a mean age of 54.05 (SD 18.75). The mean age in males was $54.12(18.89)$ and 53.96 (SD 18.57) in females. Cases distribution, stratified by age group and gender is shown in online suppl. table E-1.

Of the total number of 2,641 cases identified, 1,149 (43.5\%) were neuropathies, 953 (36.1\%) were myopathies and $539(20.4 \%)$ were neuromuscular junction disorders (fig. 3). A total of 1,380 (52.3\%) cases represented acquired NMD, which were more common in over 58-year-old groups; 1,261 (47.7\%) were inherited NMD, which were more common in 18-47 years old groups. Age-specific distribution of both acquired and inherited 
Fig. 3. Number of cases of the main groups of neuromuscular disorders. NMD = Neuromuscular disorder; $\mathrm{NMJ}=$ neuromuscular junction.

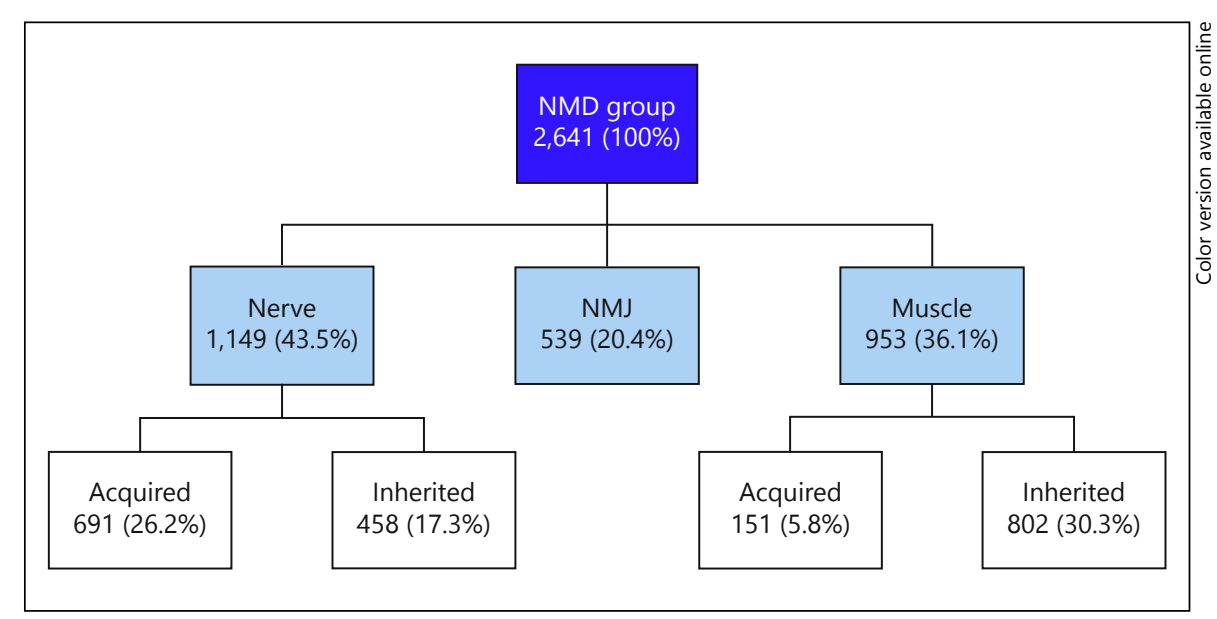

NMD is shown in online suppl. fig. E-2. Individual disorder prevalence rates will be the subject of future publications.

\section{Discussion}

In this study, a number of multiple overlapping sources were established to achieve as complete as possible case ascertainment in order to accurately estimate the countrywide prevalence of NMD. In performing this study, individual ethics applications to each hospital or source were required. Each institution had different requirements and timeframes for ethical approval rendering the process cumbersome and time consuming. This underlines the need for national research ethics committees with jurisdiction applying to multiple institutions to facilitate epidemiological research efforts of this nature.

Other obstacles encountered were the non-searchable patient databases in 2 major hospitals, which impeded case identification in the last 3 years. However, in these 2 hospitals, HIPE data crosschecked with medical charts for diagnostic verification and disease classification purposes allowed comprehensive case ascertainment. A high potential for miscoding in the HIPE system exists, highlighting the necessity, albeit laborious, to carefully crosscheck the discharge diagnosis in the medical charts. In all 4 HIPE departments analysed, the majority of cases were coded correctly apart from significant coding errors found in one institution. It was not possible to collect data from one neurology department in a public hospital and one private clinic; however, a substantial number of cases expected from these sources would have been identified through other sources such as MDI and neuropathology departments.

Neurology databases and HIPE were the two sources with the highest number of cases identified. Despite the big number overlap between these 2 sources (307), there was still a significant number of unique cases identified in these 2 sources (1,008 and 441, respectively) indicating that searching these 2 sources separately was worthwhile.

There was no significant difference between acquired and inherited NMD cases (52.8 vs. $47.2 \%$ ). As expected in adult population, inherited NMD are more common in 18-47-year-old groups and acquired NMD are more common in over 58-year-old groups. Inherited muscle diseases were the most common in the whole cohort (36.1\%) followed by acquired neuropathies (26.2\%), neuromuscular junction disorders $(20.45 \%)$ and inherited neuropathies (17.3\%).

Ascertaining cases from neurology databases included reviewing clinic/discharge letters of patients diagnosed with an NMD by a neurologist during the period 1990-2013. Clinic/discharge letters from the 1990s generally contained all the relevant and sufficient clinical information to include the patient in the study. However in $5.7 \%$ of cases of the entire cohort, the date of birth was missing because before the late 1990s it was often not recorded in clinic/discharge letters. Nevertheless, the availability of other variables (as described in Methods) yielded sufficiently accurate information to include these cases in the study. For example, some of the cases with missing date of birth have inherited NMD and at the time of the study were active members of MDI. They were thus identified in the MDI database and while the date of birth is anonymous in MDI, available details such as patient name, diagnosis and address were used for linking 
in order to prevent duplicates. Another example is that some of the discharge letters from 1990s belonged to patients who were diagnosed with GBS/AIDP and their discharge letters did not contain their date of birth either; their medical charts could not be retrieved to access their date of birth. However, by using other variables (as described above), these patients could be enrolled without duplication.

In comparison with previous similar studies (online suppl. table E-2), there are many advantages in this study. Previous studies either focused on single disorders or groups of disorders, whereas this is the first study that has captured a broad spectrum of both acquired and inherited NMD. Adult patients with NMD have many needs in common from investigation and diagnostics with neurology and neurophysiology services to expertise from cardiorespiratory services and treatment and support with experienced occupational therapy and physiotherapy services. Grouping the patients thus helps to define the burden of disease for NMD as a whole and to underline the necessity for service planning for this group of patients. Many studies focused on either clinic populations or specific geographical regions, whereas this study has focused on both neuromuscular clinic populations and the whole country population, thus avoiding referral bias to specialised centres. There are a very few adult NMD studies and some of their ascertainment techniques are similar to those used in this study (online suppl. table E-2). However, only a few studies sourced voluntary patient organisations as performed in this study. The usage of the patient support group 'Muscular Dystrophy Ireland' was an invaluable tool in sourcing many cases, which had been lost to follow-up by the neurology services. Many studies have used data from specific NMD registers, which was a more straightforward approach, but unfortunately such a registry does not yet exist in RoI. It is believed that this study will serve as the basis for setting up an NMD registry in the future. A potential disadvantage of this study is the diverse and scattered nature of the data location, resulting from the fragmented pattern of adult neurology services in the RoI. In contrast to the paediatric population, adult patients are more commonly lost to follow-up, raising the question of full capture; therefore, it becomes necessary to pursue multiple sources as delineated above. Another potential gap in ascertainment could be the exclusion of 'possible' cases currently undergoing diagnostic workup; however, we would anticipate this number to be low. Furthermore, it is possible that there are people in the community who were missed out because they were in contact with the neurology services before 1990 but not later (e.g., individuals with mild inherited neuropathies/CMT); thus, these patients would not have been captured.

In conclusion, the methods used in this study show that sourcing reliable data in the Irish health system remains challenging and requires accessing multiple sources for as complete as possible case ascertainment. Obtaining data from hospital sources is not sufficient and input from patient organisations is necessary if one is to truly capture patients with chronic diseases who are not current hospital attenders. Data recording methods in the different sources used were varied and the fragmented nature of data collection demonstrates the great need and scope to improve the existing recording standards by the development of databases and registers for these conditions.

\section{Acknowledgements}

The authors would like to thank all those who supported the study, all neurology, neurophysiology and neuropathology consultants, HIPE/Medical Records departments and Muscular Dystrophy Ireland, Ms. Margaret Cole (Statistical Consultancy, University College Cork) and Dr. Paul Corcoran (Epidemiology and Public Health, University College Cork).

\section{Disclosure Statement}

Dr. Lefter received funding via an unrestricted educational grant from Genzyme Therapeutics Ltd., and funding for travel from Muscular Dystrophy Ireland; both funders had no access to the data or writing. The authors report no conflict of interest.

References

\footnotetext{
1 Tawil R, Venance S: Neuromuscular disorders (NIP - Neurology in Practice). WileyBlackwell, October 2011, p XII.

2 Darin N, Tulinius M: Neuromuscular disorders in childhood: a descriptive epidemiological study from western Sweden. Neuromuscul Disord 2000;10:1-9.

3 Chung B, Wong V, Ip P: Prevalence of neuromuscular diseases in Chinese children: a study in southern China. J Child Neurol 2003; 18:217-219.

$\checkmark 4$ Chio A, Cocito D, Bottacchi E, et al: Idiopathic chronic inflammatory demyelinating polyneuropathy: an epidemiological study in Italy. J Neurol Neurosurg Psychiatry 2007;78: 1349-1353.

5 Foley C, Schofield I, Eglon G, Bailey G, Chinnery PF, Horvath R. Charcot-Marie-Tooth disease in Northern England. J Neurol Neurosurg Psychiatry 2012;83:572-573.
}

Methodology and Design of a National

Epidemiological Study on Adult NMD

,


6 McGrogan A, Madle GC, Seaman HE, de Vries CS: The epidemiology of Guillain-Barré syndrome worldwide. A systematic literature review. Neuroepidemiology 2009;32: 150-163.

7 Horga A, Raja Rayan DL, Matthews E, et al: Prevalence study of genetically defined skeletal muscle channelopathies in England. Neurology 2013;80:1472-1475.

8 Lucia A, Ruiz JR, Santalla A, et al: Genotypic and phenotypic features of McArdle disease: insights from the Spanish national registry. J Neurol Neurosurg Psychiatry 2012;83:322328.

9 Norwood FL, Harling C, Chinnery PF, Eagle M, Bushby K, Straub V: Prevalence of genetic muscle disease in Northern England: in-depth analysis of a muscle clinic population. Brain 2009;132:3175-3186.

$\checkmark 10$ Traynor BJ, Codd MB, Corr B, Forde C, Frost E, Hardiman O: Incidence and prevalence of ALS in Ireland, 1995-1997: a populationbased study. Neurology 1999;52:504-509.
11 Nicolaou P, Zamba-Papanicolaou E, Koutsou $\mathrm{P}$, et al: Charcot-Marie-Tooth disease in $\mathrm{Cy}$ prus: epidemiological, clinical and genetic characteristics. Neuroepidemiology 2010;35: 171-177.

12 Hughes MI, Hicks EM, Nevin NC, Patterson $\mathrm{VH}$ : The prevalence of inherited neuromuscular disease in Northern Ireland. Neuromuscul Disord 1996;6:69-73.

132011 Census of Population Interactive Tables [April 9, 2014]; Available from http://www. cso.ie/en/census/interactivetables/.

14 Van den Bergh PY, Hadden RD, Bouche P, et al: European Federation of Neurological Societies/Peripheral Nerve Society guideline on management of chronic inflammatory demyelinating polyradiculoneuropathy: report of a joint task force of the European Federation of Neurological Societies and the Peripheral Nerve Society - first revision. Eur J Neurol 2010;17:356-363

15 Asbury AK, Cornblath DR: Assessment of current diagnostic criteria for Guillain-Barre syndrome. Ann Neurol 1990;27(suppl):S21S24.
16 Emery AE: Diagnostic criteria for neuromuscular disorders, ed 2. Royal Society of Medicine, 1998.

17 Kaplan JC, Hamroun D: The 2014 version of the gene table of monogenic neuromuscular disorders (nuclear genome). Neuromuscul Disord 2013;23:1081-1111.

18 Kinali M, Beeson D, Pitt MC, et al: Congenital myasthenic syndromes in childhood: diagnostic and management challenges. J Neuroimmunol 2008;201-202:6-12.

19 Hilton-Jones D, Miller A, Parton M, Holton J, Sewry C, Hanna MG: Inclusion body myositis: MRC Centre for Neuromuscular Diseases, IBM workshop, London, 13 June 2008. Neuromuscul Disord 2010;20:142147.

20 Health Information and Quality Authority [April 9, 2014]; Available from http:// www.hiqa.ie/resource-centre/professionals/ health-information-sources/hospitalpatient-enquiry. 